\title{
The Effect of Professional and Non-Professional Courses on Mental Health among College Students
}

\author{
Naveen Pant*, Om Kant Sharma \\ Gurukula Kangri University, India \\ @naveenpantgkv@gmail.com*
}

Article Information:

Received December 13, 2020

Revised January 22, 2021

Accepted January 31, 2021

Keywords: mental health; nonprofessional course; professional course

\begin{abstract}
This study is designed to examine the impact of types of courses of study (professional/non-professional courses) on Mental Health (Anxiety, Depression, Loss of behavior or Emotional control, General positive affect, Emotional ties, or Life satisfaction) among postgraduate students. The sample is comprised of both professional and non-professional courses students of Gurukula Kangri Vishwavidyalaya, Haridwar, India. The purposive non-probability sampling method is used to select the sample of the study. The Mental Health Inventory ( $\mathrm{MHI}-38$ ) and interviews have been conducted to find out the level of mental health and its subscales among college students. As a result of the study, mental health subscales of Depression, Loss of Behavior or Emotional control, General positive affect, Emotional ties turned out to be significant functions of mental health among students, wherein these four subscales non-professional courses students found to be significantly higher than the students of the professional course. Further anxiety and life satisfaction turned out a not significant function of mental health among professional and nonprofessional course students.
\end{abstract}

\section{INTRODUCTION}

Mental health has been a focal point of study from the very beginning. The definition of the concept of mental health has been changed from time to time, because of the cultural and other differences (Rockville, 2001). Generally, mental health is a state of psychological or mental wellbeing. This study observes the role of course of study in the field of mental health.

The mental health is divided into 6 dimensions (The Mental Health Inventory, 2003) in the present study. Those are anxiety, depression, loss of behavior or emotional control, general positive affect, emotional ties, and life satisfaction. A higher score on anxiety indicates greater anxiety. A higher score on depression indicates greater depression. A higher score on the loss of behavior or emotional control indicates a greater loss of behavior or emotional control. Higher scores on General positive affect indicate greater positive affect. A high score in emotional ties means greater emotional ties. People scoring high in the dimension of Life satisfaction indicate greater life satisfaction. The objective of this study is to visualize the impact of types of course understudy (professional or non-professional courses) on mental health (anxiety, depression, loss of behavior or emotional control, general positive affect, emotional ties or life satisfaction) of postgraduate students.

Singh \& Singh (2008) studied Stress and adjustment in professional and nonprofessional students and found professional students to be poorly emotionally adjusted in comparison to non-professional, and further found professional students having more stress as

How to cite:

E-ISSN:

Published by:
Pant, N., \& Sharma, O. (2021). The Effect of Professional and Non-Professional Courses on Mental Health among College Students. Islamic Guidance and Counseling Journal, 4(1). https://doi.org/10.25217/igcj.v4i1.1243

2614-1566

Institut Agama Islam Ma'arif NU (IAIMNU) Metro Lampung 
compare to non-professional students. Sharma \& Kirmani (2015) found professional students having more symptoms of depression and anxiety than the non-professional students. Sarode \& Tendolkar (2015) Found non professional undergraduate students to be more emotionally intelligent than professional postgraduate students.

Shirazi et al. (2012) studied mental health and personality characteristics in professional and non-professional students, and found a significant relationship between mental health and personality characteristics, the agreeableness, neuroticism, and openness were found to be significant predictors of mental health. Further they found that professional and nonprofessional students have no significant difference in mental health and personality characteristics in terms of gender.

Kumar \& Bhukar (2013) found stress to be significantly higher among girls in comparison to boys of their profession. The coping strategy was found to be higher in boys than girls in terms of their respective professions, but Physical Education girls were found to have higher coping strategy than boys and girls of Engineering, finally, they concluded Physical Education student to better in coping strategy than engineering students. But Menka (2016) found neutral results stating no significant difference between professional and non professional students in terms of anxiety, and also found no significant difference between male professionals and male non professionals in terms of anxiety.

Joshi \& Garg (2013) found a significant difference between males and females in mental health. And further found a significant difference between Length of service for more than 10 years and length of service for less than five years. Nisa (2013) found extraversion to be a significant predictor for positive self-evaluation; openness to be a significant predictor for the perception of reality, agreeableness for the integration of personality. Ashwini \& Barre (2014) found a significant difference in stress experience among students. And further found a significant difference between arts and science students in terms of adjustment areas, a significant difference between boys and girls in terms of academic performance and a significant difference between arts and science in terms of academic performance. Sharma (2014) found that, among adolescents, gender and stream have a significant interaction effect on depression. Girls were higher on depression as compared to boys, and arts students were found to be highly depressed as compared to science and commerce students. Further, he got no difference among science, arts, and commerce stream boys on depression. Among girls of science, arts and commerce streams a significant difference was found in terms of depression, favouring arts girls. Finally, he indicated gender and stream to be effective in terms of depression in adolescents.

Kumari \& Jain (2014) Found a relationship between examination stress and anxiety among college students and arts students were found having highest stress and anxiety during examination in compare to science students. But Singh \& Singh (2008) found contrary results they found Professional students having more stress as compared to non-professional students. Seth \& Asudani (2014) found that the professional courses students have higher level of suicidal ideation than the non-professional course students. Arora (2015) Found male students of professional courses showing slightly higher degree of positive adaptability regarding changes taking place about position of women in society than female students. On other hand females of Non-professional students are slightly high than men regarding adapting positive changes.

In a recent study, Pant \& Srivastava (2019) found a significant positive relationship between spiritual intelligence and mental health among arts and Science students and found no significant difference between art and science students on mental health. Shirazi \& Khan (2013) found no significant difference among professional students' life satisfaction in terms of residence, gender. For nonprofessional students' life satisfaction they found significant difference in terms of residence and gender. 


\section{Rationale of the Current Study}

As this study is conducted with these mental health dimensions (anxiety, depression, loss of behavior or emotional control, general positive affect, emotional ties, and life satisfaction) in Indian context among professional and no professional students is the core part of the study. Significant part of the study is that, this is the first of its kind conducted in Indian Gurukula setting for knowing the mental health levels, differences and challenges among college students having professional and non-professional stream in their education.

\section{Hypotheses}

Type of course understudy (professional or non-professional) will influence mental health (anxiety, depression, loss of behavior or emotional control, general positive affect, emotional ties, and life satisfaction) of postgraduate students. Author also registered six subhypotheses.

1. There will be no significant difference between the levels of anxiety in postgraduate students of professional and non-professional courses.

2. There will be no significant difference between the levels of depression in postgraduate students of professional and non-professional courses.

3. There will be no significant difference between the levels of loss of behavior or emotion control in postgraduate students of professional and non-professional courses.

4. There will be no significance difference between the levels of general positive affect in postgraduate students of professional and non-professional courses.

5. There will be no significant difference between the levels of emotional ties in postgraduate students of professional and non-professional courses.

6. There will be no significant difference between the levels of life satisfaction in postgraduate students of professional and non-professional courses.

\section{METHODS}

\section{Research Design}

This study is based on non-experimental research design. Adequate techniques were applied to control the intervening or extraneous variance of the study. Such as 1) subjectrelevant variables (a) age should 21-25 years (b) education should post graduation. environment, the subjects in the study were analyzed in a comfortable environment. Care was taken to free them from any kind of stress of fatigue as for as possible. Relevant and related information about the test was provided to the participants.

Type of courses under study (professional or non-professional) is the independent measure in the research. Mental Health is dependent measure in the study. It has six dimensions 1) anxiety, 2) depression, 3) loss of behavior or emotional control, 4) general positive affect, 5) emotional ties, and 6) life satisfaction.

\section{Participants}

In this study, the purposive non-probability sampling method for drawing sample has been used. The final sample of 60 students (30 professional and 30 non-professional courses) has been cautiously drawn. The participants of Mental Health has been drawn from Gurukula Kangri Vishwavidyalaya (Deemed University), Haridwar. The elements of the universe are both postgraduate students of professional (MBA, MCA etc.) and non-professional (MA etc.) courses studying at university campus. Their age ranges between $21-25$ years. They all are males. 


\section{Instruments}

The following tools were used for collection of the data. 1) Personal bio-data sheet, this was a front page of the questionnaire and has the options for the demographic entry. It included instructions, name (not compulsory to be filled), gender, age and educational stream. 2) Interview sessions were conducted to collect the necessary details about the Mental Health. 3) The Mental Health Inventory (MHI-38), The Mental Health Inventory (2003)'s all of the $38 \mathrm{MHI}$ items, except two, are scored on a six-point scale (range 1-6). Items 9 and 28 are the exception, each scored on a five-point scale (range 1-5). The questionnaire provides scores for 6 separate Mental Health Subscales. These are anxiety, depression, loss of behavior or emotional control, general positive affect, emotional ties, and life satisfaction. Higher scores on three subscales indicate positive states of mental health (general positive affect, emotional ties, life satisfaction); higher scores on the other three subscales indicate negative states of mental health (anxiety, depression, loss of behavioral or emotional control).

\section{Procedures}

Data collection procedure was adopted with relevant precautions. All participants were taken from the Haridwar (one of the most famous religious city of the world). Detailed information about the Mental Health was gathered from interviewing and 60 cases of post graduate students: 30 professional and 30 non-professional courses were selected. Mental Health Inventory (MHI) was cautiously administered on all 60 selected participants.

\section{Data Analysis}

Each score was tabulated for finding the nature of the test scores of all variables under consideration. Mean, Median, Standard deviation, skewness, kurtosis, and T-Test is applied to find the results and a significance of the difference at the level of .01 and .05 among all the variables.

\section{RESULTS AND DISCUSSION}

\section{Results}

Based on the results of data analysis, the Table 1 showed the demographical Table 1. The demographical information of participants

\begin{tabular}{llll}
\hline Category of Students & Gender & Age in years & $\mathrm{N}$ \\
\hline Professional Course & Male & $21-25$ & 30 \\
Non- Professional Course & Male & $21-25$ & 30 \\
\hline
\end{tabular}

Table 2. The differences between each variables in professional and non-professional courses

\begin{tabular}{llllllll}
\hline Variables & Category & $\mathrm{M}$ & SD & SED & df & t-value & $\mathrm{p}$ \\
\hline AN & Pro & 24.3 & 6.73 & 1.62 & 58 & .90 & $>.05$ \\
& Non & 25.76 & 5.85 & & & & \\
DE & Pro & 9.8 & 3.67 & 0.87 & 58 & 2.26 & $<.05$ \\
& Non & 11.7 & 3.02 & & & & \\
LC & Pro & 18.96 & 5.29 & 1.26 & 58 & 2.23 & $<.05$ \\
& Non & 23.26 & 4.48 & & & & \\
GP & Pro & 38.06 & 9.71 & 2.05 & 58 & 2.11 & $<.05$ \\
& Non & 42.40 & 5.66 & & & & \\
ET & Pro & 8.40 & 2.52 & .52 & 58 & 2.21 & $<.05$ \\
& Non & 9.56 & 1.38 & & & & \\
LS & Pro & 4.40 & .89 & 0.25 & 58 & 1.58 & $>.05$ \\
& Non & 4.00 & 1.05 & & & & \\
\hline
\end{tabular}

Pro $=$ Professional, Non $=$ Non-Professional

$\mathrm{AN}=$ Anxiety, $\mathrm{DE}=$ Depression, $\mathrm{LC}=$ Loss of behavior or emotion control, $\mathrm{GP}=$ General positive affect, ET = Emotional ties, LS = Life Satisfaction 
information of participants. Table 2 showed that there is no signifincant difference between two means of anxiety and life satisfaction levels in postgraduate students of professional and non-professional courses. Besides, there is a significant difference between two means of Depression, Loss of behavior or emotion control, General positive affect, and Emotional ties levels in postgraduate students of professional and non-professional courses.

\section{Discussion}

As it is previously discussed higher scores on three subscales indicate positive states of mental health (general positive affect, emotional ties, life satisfaction); higher scores on the other three subscales indicate negative states of mental health (anxiety, depression, loss of behavioural or emotional control). Results of the present study show that professional and non-professional courses of postgraduate students determine the difference in the 4 dimensions; level of depression, loss of behavior or emotional control, general positive affect, and emotional ties, but does not determine the 2 dimensions; level of anxiety and life satisfaction. Where past studies are both in favour and contrary of present studys results.

No significant difference has been found between the levels of anxiety in postgraduate students of professional courses and non-professional courses. In this study, results imply that there would be no significant difference in postgraduate students of professional and nonprofessional courses regarding the level of anxiety. It may be due to postgraduate students of both study types easily face the almost similar challenges of everyday life based on their own needs. Same as Menka (2016) found neutral results stating no significant difference between professional and non professional students in terms of anxiety, and also found no significant difference between male professionals and male non professionals in terms of anxiety.

A significant difference has been found between the levels of depression in postgraduate students of professional courses and non-professional courses. This result shows that depression level of postgraduate students of both study types differs significantly. The level of depression has been found more in postgraduate students of non-professional courses than professional courses. In sum, the findings in this study were in line with the findings of past research. Sharma (2014) found the similar results that, among adolescents, gender and stream have a significant interaction effect on depression. Arts students were found to be highly depressed as compared to science and commerce students.

A significant difference has been found between the levels of loss of behavior or emotional control in postgraduate students of professional and non-professional courses: this result implies that loss of behavior or emotional control level of postgraduate students of both study types differs significantly. The level of loss of behavior or emotional control has been found more in postgraduate students of non-professional courses than professional courses. In support of results Kumari \& Jain (2014) found arts students having highest stress and anxiety during examination in compare to science students. The contrary results were found by Singh \& Singh (2008) where they found professional students to be poorly emotionally adjusted in comparison to non-professional. Further found professional students having more stress as compare to non-professional students.

A significant difference has been found between the levels of general positive affect in postgraduate students of professional and non-professional courses: this result implies that general positive affect level of postgraduate students of both study types differs significantly. The level of general positive affect has been found more in postgraduate students of nonprofessional courses than professional courses. Singh \& Singh (2008) found the similar results; they got professional students to be poor in emotional adjustment in comparison to non-professional.

A significant difference has been found between the levels of emotional ties in postgraduate students of professional and non-professional courses: this result implies that 
emotional ties level of postgraduate students of both study types differs significantly. The level of emotional ties has been found more in postgraduate students of non-professional courses than professional courses. In support of the results of the present study Sarode \& Tendolkar (2015) found non professional undergraduate students to be more emotionally intelligent than professional postgraduate students

No significant difference has been found between the levels of life satisfaction in postgraduate students of professional and non-professional courses: this result shows the life satisfaction level of postgraduate students of both study types does not differ significantly. Same as pant and srivastava (2019) found no significant difference between art and science students on mental health. Finally, Pant \& Srivastava (2015) found no significant difference between art and science students on quality of life.

\section{Limitations and Suggestions}

Being behavioral science research the present research also had some limitations. The main limitations of this study are: The sample may be larger for the purpose and may be drawn from various colleges, institutes, or universities of all territories of Uttarkhand State, India. Any method of probability sampling process may be used instead of a purposive nonprobability method that has been used in the present study. Other independent variables as socioeconomic status, gender, and quality of life may be studied in further research.

\section{Implications}

The study will be helpful in determining the behavior and techniques among professional and nonprofessional courses' students, with especial focus on nonprofessional. Because present study's all variance indicates towards nonprofessional students whether in favour and oppose, as Ahmad \& Ahamad (2016) also found the non-professional students to be more anxious in compression to professional students in both gender. The present study suggests the need of mental health services and interventions for college going students, as researches indicating the various mental health challenges among students. There on one side the results indicate the causes and challenges of mental health among students of professional and non-professional course, on other side provides a direction for the new research and therapeutic approach in light of past favourable and contradictory studies. The study will be helpful and center of attraction among, school counselors, psychologists, researchers, parents etc.

The present study finally suggests mindfulness-based interventions for the college students as McConville et al. (2017) suggest on basis of their research on health profession students that it is helpful in decreasing stress, anxiety, and depression, and improves empathy, mood, self-efficacy and mindfulness, and mindfulness-training could be easily adapted among these students.

\section{CONCLUSSION}

The six Mental Health subscales are important for scientific study. They characteristically remain a function of Mental Health. For instance, Mental Health subscales of Depression, Loss of Behavior or Emotional control, General positive effect, Emotional ties turned out to be significant functions and anxiety and life satisfaction turned out a not significant function of Mental Health. This study explores that postgraduate students of professional and non-professional courses promote the same experience with anxiety and life satisfaction. Feels depression, loss of behavior oremotion control, general positive affect and emotion ties differently as the basis of their profession. The area of the investigation remains highly prospective and new. Results of more sophisticated researches in the area would pave the way to new vistas of research. 


\section{ACKNOWLEDGMENTS}

Authors are thankful to all the students who helped them as being the participants of the study, and owe thanks to those who helped them directly and indirectly making this study come to the end.

\section{AUTHOR CONTRIBUTIONS STATEMENT}

OK designed study, collected data, made data analysis and got all necessary permissions for the study. NP provided past review of literature, converted the unstructured study into structured one with necessary addition, and prepared the manuscript.

\section{REFERENCES}

Ahmad, Z. \& Ahmad, E. (2016). A comparative study on anxiety between professional and non-professional graduate level students. International Journal of Applied Research, 2(6), 456-460. Google Scholar

Arora, M. (2015). A comparative study between professional and non-professional course students: Social Adaptability. International Journal of Humanities \& Social Science Studies, 1(5), 26-31. Google Scholar

Ashwini, R., \& Barre, V. P. (2014). Stress and adjustment among college students in relation to their academic performance. Indian Journal of Health and Wellbeing, 5(3), 288- 292. Google Scholar

Joshi, R., \& Garg, J. M. (2013). Mental health in reference to length of service among males and females academicians. International Journal of Research in Commerce \& Management, 4(11), 38- 40. Google Scholar

Kumar, S., \& Bhukar, J. P. (2013). Stress level and coping strategies of college students. Journal of Physical Education and Sports Management, 4(1), 5-11. https://doi.org/10.5897/JPESM12.001

Kumari, A., \& Jain, J. (2014). Examination stress and anxiety: a study of college students. Global Journal of Multidisciplinary Studies, 4(1), 31-40. Google Scholar

Menka, M. (2016). A study of anxiety of professional and non professional college students. International Journal of Science and Research, 5(12), 1489-1491. Google Scholar

McConville, J., McAleer, , R., \& Hahne, A. (2017). Mindfulness training for health profession students- the effect of mindfulness training on psychological well-being, learning and clinical performance of health professional students: a systematic review of randomized and non-randomized controlled trials. Explore, 13(1), 26-45. https://doi.org/10.1016/j.explore.2016.10.002

Nisa, M. (2013). Study of personality factors and family problems as determinant of mental health amongst college students. International Journal of Advancements in Research \& Technology, 2(11), 22- 32. Google Scholar

Pant, N. \& Srivastava, S. K. (2015). Spiritual intelligence, gender and educational background as related to the quality of life of college students. Indian Journal of Positive Psychology, 6(3), 226-232. Google Scholar

Pant, N. \& Srivastava, S. K. (2019). The impact of spiritual intelligence, gender and educational background on mental health among college students. Journal of Religion and Health, 58(1), 87-108. https://doi.org/10.1007/s10943-017-0529-3

Rockville, R. (2001). Office of the Surgeon General (US); Center for Mental Health Services (US); National Institute of Mental Health (US). Mental Health: Culture, Race, and Ethnicity: A Supplement to Mental Health: A Report of the Surgeon General. Rockville (MD): Substance Abuse and Mental Health Services Administration (US); 2001 Aug. Chapter 2 Culture Counts: The Influence of Culture and Society on Mental Health. Google Scholar 
Sarode, R., \& Tendolkar, V. (2015). Comparative study of emotional intelligence among professional \& non-professional college students. Journal of Datta Meghe Institute of Medical Sciences University, 10(4), 214-219. https://doi.org/10.4103/jdmimsu.jdmimsu_175_19

Seth, M. R., \& Asudani, V. H. (2014). Suicidal ideation in students pursuing professional and non professional courses. Asian Academic Research Journal Of Multidisciplinary, 1(25). 195-209. Google Scholar

Sharma, P. \& Kirmani, M. N. (2015). Exploring depression \& anxiety among college going students. International Journal of Science and Research, 4(6), 528-532. Google Scholar

Sharma, V. (2014). Effect of gender and stream on depression among adolescents. International Journal of Education and Psychological Research, 3(2), 46- 49. Google Scholar

Shirazi, M., Khan, M. A., \& Ansari, M. F. A. (2012). Mental health in relation to personality characteristics among professional and non-professional students. Journal of Arts, Science \& Commerce, 3(1), 8- 15. Google Scholar

Shirazi, M., \& Khan, M. A. (2013). Life satisfaction among professional and non-professional students in India. International Journal of Applied Psychology, 3(4), 109-113. https://doi.org/10.5923/j.ijap.20130304.03

Singh, A. \& Singh, S. (2008). Stress and adjustment among professional and non professional students. Industrial Psychiatry Journal, 17(1), 26- 27. Google Scholar

The Mental Health Inventory. (2003). Mental Health national outcomes and case mix collection: Overview of clinician-rated and consumer self-report measure, Version 1.50. Canberra: Department of Health and Aging. Google Scholar

Copyright holder :

(C) Pant, N., \& Sharma, O. (2021)

First publication right :

Islamic Guidance and Counseling Journal

This article is licensed under:

CC-BY-SA 